\title{
Suporte de pares como estratégia de recovery e a experiência dos redutores de danos da rede pública de saúde mental de belo

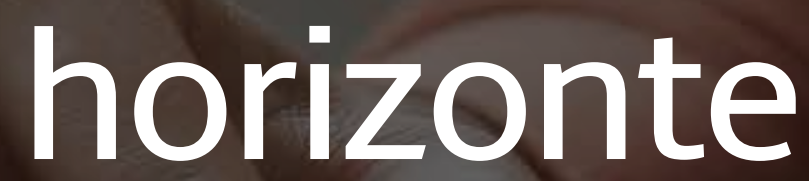

apoyo de pares como estrategia de recuperación y experiencia de reductores de daños de la red de salud pública de belo horizonte Couple support as a strategy for the recovery and experience of harm reducers of the belo horizonte public health network

Olívia Malheiro Siqueira

Caroline Oliveira Romao Ana Sophia Torres de Oliveira

Breno Rodrigo Diniz Ângelo de Azeredo Coutinho

Louise Arcanjo de Paula 
Couple support as a strategy for the recovery horizonte public health network

Título: $\quad$ Apoyo de pares como estrategia de recuperación y experiencia de reductores de daños de la red de salud pública de belo horizonte

Título: Suporte de pares como estratégia de recovery e a experiência dos redutores de danos da rede pública de saúde mental de belo horizonte

Alt Title / Título alternativo / Título alternativo:

[en]: $\quad$ Couple support as a strategy for the recovery and experience of harm reducers of the belo horizonte public health network

[es]: $\quad$ Apoyo de pares como estrategia de recuperación y experiencia de reductores de daños de la red de salud pública de belo horizonte

[pt]: O suporte de pares como estratégia de recovery: uma construção coletiva a partir da experiência dos redutores de danos da rede pública de saúde mental de belo horizonte

Author (s) / Autor (es) / Autor (es)

Malheiro Siqueira, Oliveira Romao, Torres de Oliveira, Diniz, de Azeredo Coutinho \& de Paula

Keywords / Palabras Clave / Palavras chave:

[en]: Recovery, peer support, harm reduction, experience report, mental health, peer support in Brazil

[es]: $\quad$ Recovery, soporte de pares, reductor de daños, informe de experiencia, Salud mental, apoyo de pares en Brasil

[pt]: Recovery, suporte de pares, redução de danos, relato de experiência, saúde mental, suporte de pares no Brasil

Proyecto / Project:

No Reporta

Financiación / Funding:

No Reporta

Submited: $2020 / 08 / 29$

Acepted: $\quad 2020 / 10 / 31$ and experience of harm reducers of the belo

\section{Resumen}

La recuperación es un concepto que surgió en los años 70 para designar el proceso en el que las personas que experimentan un problema de salud mental son capaces de manejar dicha experiencia de una manera que les permita tener una vida significativa, con un sentido positivo de pertenencia a la comunidad. Una de las estrategias de recuperación es el apoyo entre pares, que consiste en la interacción, con un enfoque en brindar apoyo, entre personas que viven con problemas de salud mental y otras personas que atraviesan situaciones similares. Si bien la contratación de pares de apoyo en Brasil es aún incipiente, se argumenta en este artículo que la experiencia de reducción de daños, al menos tal como se configura en la red pública de salud mental en Belo Horizonte, consiste en un modelo brasileño cercano al representado para el apoyo de pares en otros países. Así, el presente trabajo tuvo como objetivo reportar la experiencia de tres reductores de daños en Belo Horizonte, para investigar el potencial de reducción de daños como estrategia de recuperación.

\section{Abstract}

Recovery is a concept that emerged in the 1970s to designate the process in which people who experience a mental health problem become able to manage such an experience in a way that makes it possible for them to have a meaningful life, with a positive sense of belonging to the community. One of the recovery strategies is peer support, which consists of the interaction - with a focus on providing support - between people who live with mental health conditions and other people who go through similar situations. Although the hiring of peer supporters in Brazil is still incipient, it is argued in this article that the experience of harm reduction, at least in the way it is configured in the public mental health network in Belo Horizonte, consists of a Brazilian model close to the one represented by peer support in other countries. Therefore, the present study aimed to report the experience of three damage reducers in Belo Horizonte, to ascertain the potential of damage reduction as a recovery strategy.

\section{Resumo}

Recovery é um conceito que surgiu na década de 70 para designar o processo no qual pessoas que vivenciam algum problema de saúde mental se tornam capazes de manejar tal experiência de maneira que seja a elas possibilitada uma vida significativa, com senso positivo de pertencimento à comunidade. Uma das estratégias do recovery é o suporte de pares, que consiste na interação - com foco em provisão de apoio - entre pessoas que convivem com condições de saúde mental e outras pessoas que passam por situações semelhantes. Apesar de ainda incipiente a contratação de apoiadores de pares no Brasil, argumenta-se neste artigo que a experiência de redução de danos, ao menos da forma como se configura na rede pública de saúde mental de Belo Horizonte, consiste num modelo brasileiro próximo daquele representado pelo suporte do pares em outros países. Sendo assim, o presente trabalho objetivou relatar a experiência de três redutores de danos em Belo Horizonte, para averiguar as potencialidades da redução de danos como estratégia de recovery.

\section{Citar como:}

Malheiro Siqueir, O., Oliveira Romao, C., Torres de Oliveira, A. S., Diniz, B. R., de Azeredo Coutinho, Â. \& de Paula, L. A. (2021). O suporte de pares como estratégia de recovery: Uma construção coletiva a partir da experiência dos redutores de danos da rede pública de saúde mental de belo horizonte. Revista Iberoamericana de Psicología , 14 (2), 49-57. https://reviberopsicologia.ibero.edu.co/article/ view/2204
Caroline Oliveira Romao, Med sp

BIO:

Médica, con residencia en Psiquiatría

City I Ciudad:

Belo Horizonte-MG [br]

e-mail:

carol.o.romao@hotmail.com
Goiânia-GO, [br]

e-mail:

oliviapsiquiatra@gmail.com

Breno Rodrigo Diniz, Lic-psi

ORCID: $\quad$ 0000-0003-1089-504X

City I Ciudad:

Guarapari-ES, Brasil

No

e-mail:

brenorodrigord@gmail.com

Ana Sophia Torres de Oliveira, Med

ORCID: $\quad$ 0000-0002-4790-7588

Source I Filiacion:

Cersami noroeste

BIO

Médica

City I Ciudad:

Belo Horizonte-MG [br]

e-mail:

anasophiatorres@gmail.com
Ângelo de Azeredo Coutinho

ORCID: $\quad$ 0000-0001-9939-5513

City I Ciudad:

Belo Horizonte-MG, Brasil

e-mail:

angelocoutinho@hotmail.com

\section{Louise Arcanjo de Paula}

ORCID: $\quad$ 0000-0003-1625-1560

City I Ciudad:

Belo Horizonte-MG, Brasil

e-mail:

louisedubrazel@gmail.com 


\section{Suporte de pares como estratégia de recovery e a experiência dos redutores de danos da rede pública de saúde mental de belo

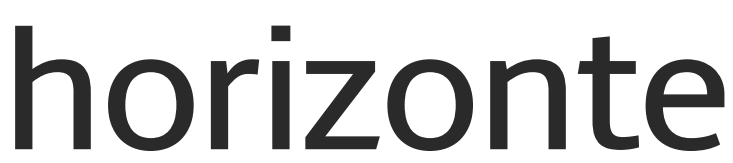

apoyo de pares como estrategia de recuperación y experiencia de reductores de daños de la red de salud pública de belo horizonte Couple support as a strategy for the recovery and experience of harm reducers of the belo horizonte public health network

Olívia Malheiro Siqueira

Caroline Oliveira Romao Ana Sophia Torres de Oliveira Breno Rodrigo Diniz Ângelo de Azeredo Coutinho

Louise Arcanjo de Paula

Até a década de 1980, hospitais psiquiátricos e asilos eram os principais locais de tratamento para pessoas que convivessem com algum problema de saúde mental grave no Brasil. Com maior intensidade a partir da segunda metade da década de 1970, a Reforma Psiquiátrica instituiu uma nova política de saúde mental, que teve como um de seus principais resultados o desenvolvimento dos Centros de Atenção Psicossocial (CAPS). Esse processo marcou a transição de um modelo hospitalocêntrico para um de saúde mental comunitária, com considerável redução dos leitos psiquiátricos e implantação de serviços substitutivos (Onocko-Campos et al., 2012). Ainda é um desafio, contudo, incorporar, na prática, o cuidado centrado no desejo da pessoa com transtorno mental (Costa, 2017). De forma concomitante à Reforma Psiquiátrica no Brasil, surgiu nos Estados Unidos um movimento de ex-pacientes que deu origem ao conceito de recovery, que então estabeleceu-se como um novo paradigma em Saúde Mental (Baccari et al., 2015).

De acordo com o Department of Mental Health and Addiction Service (DMHAS) (2002), recovery designa o processo pelo qual pessoas que vivenciam algum problema de saúde mental se tornam capazes de manejar seu transtorno mental e/ou seu transtorno de uso de substâncias, de forma que seja possível ter uma vida significativa e com senso positivo de pertencimento à comunidade. O movimento que culminou na noção de recovery reconhece que pessoas com um diagnóstico grave em saúde mental têm o direito de ter uma vida comunitária plena. (Davidson, 2016). Assim, recovery 
não é sinônimo de cura e nem simplesmente envolve o retorno a um estado pré-mórbido, ao contrário, é um processo de vida que envolve a própria experiência do adoecimento, a aceitação desta condição e a incorporação de novos sentidos para experiência, a superação do estigma, o exercício da cidadania e a responsabilização pela vida. (Davidson, 2003, p. 44)

Dentre as estratégias do recovery figura o suporte de pares ou peer support -, uma prática que se baseia na concepção de que pessoas que convivem com problemas de saúde mental e/ou abuso de substâncias podem oferecer, de forma voluntária ou remunerada, apoio a pessoas que passam por condições semelhantes (Davidson et al., 2008). O suporte de pares cultiva como princípios apoio e respeito mútuos, o compartilhamento de responsabilidades e a alteridade.

Já foram documentados diversos benefícios associados ao suporte de pares para pessoas com transtornos mentais - tanto para aquelas que fornecem apoio quanto para aquelas que o recebem. Dentre esses benefícios, cabe citar: desenvolvimento de conexões interpessoais; ampliação da visão de mundo; mutualidade; e movimento em direção a novas conquistas. Também considera-se como benefício a promoção da esperança, da autoestima, do empoderamento, da integração social e da qualidade de vida. Além disso, o suporte de pares viabiliza o envolvimento das pessoas nos serviços necessários para seu tratamento e incentiva os usuários a assumir o protagonismo em seus processos de recovery. (Davidson, 2018).

A literatura também sustenta que a implementação do suporte de pares pode melhorar a eficácia dos serviços de saúde mental e reduzir os custos para a rede de saúde, bem como aumentar a satisfação dos usuários com os serviços. Há evidências de que o suporte de pares melhora o autocuidado dos pacientes e reduz gastos com atendimentos em urgência, uma vez que diminui as internações e os sintomas psiquiátricos (Campos et al., 2014). Além disso, o trabalho com suporte de pares também oferece benefícios específicos aos indivíduos que ofertam o suporte, tais como o desenvolvimento de habilidades profissionais, a remuneração - em alguns casos -, e a possibilidade de reconhecer as próprias necessidades materiais e sociais, operação que é, por vezes, dificultada a esses indivíduos, uma vez que fazem parte de um grupo que é tradicionalmente preterido pelo mercado de trabalho (Costa et al., 2017).

Estima-se que já existam nos Estados Unidos mais de dez mil apoiadores de pares, e a prática é crescente em todo o mundo (Davidson, 2018). Tal como indica Costa (2017), a implementação desta prática é um trabalho complexo, mas possui potencial para promover mudanças culturais significativas nos serviços de saúde mental. No Brasil, o suporte de pares ainda é pouco empregado. Contudo, ao analisar de forma mais detida a experiência brasileira de redução de danos, pode-se colocar como hipótese que esse tipo de atuação, em algumas circunstâncias - mais especificamente, da forma como é desenvolvida em Belo Horizonte -, pode se apresentar como um modelo pontual de aplicação do suporte de pares como técnica de recovery.

Segundo Silveira (2016, p. 111), redução de danos é "uma política pública e um conjunto de princípios e práticas que objetiva ao uso de drogas por parte do usuário, da forma menos danosa possível para ele e seu entorno social". Ainda segundo o autor, a redução de danos

difere das propostas de combate às drogas focadas na redução de oferta e de demanda, uma vez que as ações buscam uma atenção em saúde que seja equânime, universal e integral, em que a finalidade não é a abstinência total, e sim o autocuidado e a garantia do direito de acesso a serviços de saúde qualificados, disponíveis e necessários. (Silveira, 2016, p. 111)

A redução de danos chegou ao Brasil em 1989, com uma iniciativa de distribuição de seringas pela Secretaria da Saúde de Santos (Passos \& Souza, 2011). Na época, a cidade era a que mais contava casos de Aids no país; muitos deles, ocasionados pela partilha de seringas. A despeito do caráter dessa primeira intervenção de redução de danos no Brasil, contudo, "os redutores brasileiros nunca se limitaram às trocas de seringas ou à prevenção e ao tratamento da Aids" (Petuco, 2014). Com efeito, desde seu aparecimento no país, a redução de danos se capilarizou como prática, variando seu campo de atuação e conquistando espaço institucional mais sólido - ainda que historicamente tenha enfrentado obstáculos (Petuco, 2011). Um exemplo da consolidação da redução de danos no país é a presença dessa técnica como uma das estratégias constituintes da Política Nacional de Atenção Integral a Usuários de Álcool e Outras Drogas.

Estabelecida pela Portaria nº 3.088 (2011) do Ministério da Saúde, a Política Nacional de Atenção Integral a Usuários de Álcool e Outras Drogas preconiza ações de redução de danos. Dentre elas, está a atuação do redutor de danos na Rede de Atenção Psicossocial (RAPS) do Sistema Único de Saúde (SUS). Na cidade de Belo Horizonte, os redutores de danos são remunerados por Organizações Não Governamentais (ONGs) que possuem convênio com o município e são alocados nos Centros de Referência à Saúde Mental de Álcool e Outras Drogas (CERSAM AD), que constituem serviços de atenção às pessoas com transtornos relacionados ao uso de álcool e outras drogas; nos Centros de Referência à Saúde Mental Infantil (CERSAMI), que constituem serviços de atendimento à saúde mental da criança e do adolescente em urgência psiquiátrica; e nos chamados Consultórios de Rua, constituídos por um grupo de trabalhadores que percorre a cidade em unidade móvel, abordando pessoas em uso de álcool, crack e outras drogas.

A redução de danos se configura como uma estratégia de política pública que sofre constantes ataques à sua legitimidade (Petuco, 2011; Nardi \& Rigoni, 2005; Antunes, 2019). Mas o caso dessa política específica não é exceção: desde a implementação da Reforma Psiquiátrica - resultado do movimento mais amplo representado pela Luta Antimanicominal (Petuco, 2011) - há dificuldade na consolidação de abordagens terapêuticas que não firam a autonomia dos atendidos. Tendo isso em vista, o objetivo desse estudo é relatar a experiência de redutores de danos na cidade de Belo Horizonte, a fim de verificar se a redução de danos em Belo Horizonte pode ser classificada como uma ação de suporte de pares - prática ainda incipiente no Brasil, mas globalmente reconhecida como legítima. Caso evidenciada tal afinidade, se faz pertinente a consideração da redução de danos a partir de um novo status, a saber: como ponta de lança da introdução de práticas de recovery no país.

\section{Métodos}

A metodologia empregada para dar conta do objetivo estipulado baseou-se naquela pertinente a um relato de experiência, a saber: a descrição de relatos. Assim, foram coletadas informações por meio da sistematização dos relatos de três dos coautores do presente artigo, todos redutores de danos. Tendo em vista que as metodologias qualitativas são as mais adequadas para entender como um fenômeno interfere na vida das pessoas (Davidson et al., 2008), buscou-se analisar qualitativamente as colocações de cada um dos contribuintes, para que se tornasse possível traçar um breve panorama do 
funcionamento da experiência de redução de danos em Belo Horizonte e compará-la ao suporte de pares como estratégia de recovery. Os relatos foram coletados entre janeiro e julho de 2020, inicialmente em encontros presenciais, e posteriormente via plataforma Zoom, devido à recomendação de distanciamento social relacionada à pandemia da COVID-19. A análise dos relatos de experiência permitiu identificar e sistematizar experiências singulares e as diferentes trajetórias de cada indivíduo envolvido no processo de redução de danos, notadamente no que diz respeito à construção de uma vida significativa e satisfatória.

\section{Resultados}

Com base nos relatos colhidos para este trabalho, foi possível afirmar que embora não haja uma disposição expressa para que o agente redutor de danos seja alguém que fez ou faz uso de substâncias, verificou-se que os serviços de Belo Horizonte que implementaram sua contratação, em sua maioria, optaram por profissionais que já passaram pela experiência do uso de álcool e outras drogas. Assim, a partir do que foi relatado, pode-se afirmar que há, como pré-requisito para a atuação como redutor de danos na rede pública de atenção à saúde mental (RAPS) de Belo Horizonte, que o candidato tenha vivido experiências pessoais em saúde mental - sobretudo experiências relacionadas ao uso nocivo de substâncias. Sobretudo, os redutores de danos nesta cidade devem ser, preferencialmente, pessoas que se encontram em processo de recovery. Logo, os redutores de danos podem, a depender do aparecimento de elementos complementares, ser considerados apoiadores de pares cujo papel é auxiliar outras pessoas que estejam em sofrimento mental relacionado ao uso abusivo de álcool e outras drogas.

Dessa forma, procurou-se analisar os relatos de experiência a partir de dois eixos principais, considerados os pilares do suporte de pares, para avaliar a presença de elementos complementares que intensifiquem as afinidades entre a atuação como redutor de danos em Belo Horizonte e a atividade de suporte de pares como estratégia de recovery. Assim, foram observadas: (1) a influência do trabalho na própria trajetória de recovery do redutor de danos e (2) a influência da própria vivência em saúde mental do redutor de danos no trabalho. Em cada um desses eixos, foram observados diferentes elementos temáticos que, por sua vez, balizaram a análise realizada. No que diz respeito ao eixo (1), destacaram-se colocações envolvendo rotina, emprego e autoestima, bem como adesão ao próprio tratamento. Já no que se refere ao eixo (2), apareceram temas relativos a linguagem, identificação e empatia, ao conhecimento próprio sobre os efeitos e condução do tratamento e à própria experiência como fonte de esperança. Os trechos dos relatos que correspondem a essa divisão temática serão expostos a seguir.

\section{Influência do trabalho na própria trajetória de Recovery}

\subsection{Rotina, emprego e autoestima}

O suporte de pares é uma via de mão dupla que também é proveitosa para a jornada de recovery dos próprios profissionais apoiadores, ao permitir que tenham um trabalho pessoalmente motivado, com significado para o sujeito (Davidson et al., 2018, p. 11). Todos os redutores de danos entrevistados relataram ganhos relacionados à importância de uma rotina de trabalho estruturada; também foram relatados ganhos de autoestima associados à sua posição como agentes ativos de transformação social, o que contribui para os seus processos pessoais de recovery.

\begin{abstract}
"E por outro lado o meu trabalho me garante na saúde mental (...) Eu tenho minha rotina, eu tenho que trabalhar todos os dias. Isso me organiza porque além de trabalhar eu estudo, faço outras coisas, ando de skate, faço todas as coisas que eu tenho que fazer em tempo hábil e isso se dá muito pelo meu trabalho, porque tipo assim: não é só ir lá e pegar um dinheiro todo mês, trabalhar o mês todo para ir lá e pegar um dinheiro todo mês, mas sim poder fazer a diferença ali numa conversa, num acolhimento. Você pode 'trocar uma ideia' com uma pessoa, pelo menos na minha posição de redução de danos, eu posso fazer uma diferença, eu posso mostrar outros caminhos, talvez, para uma pessoa que esteja ali 'na pior', precisando de uma ajuda”. (Relato do participante 1).
\end{abstract}

No que diz respeito às possibilidades de que o apoiador de pares tenha seu trabalho afetado por seus problemas de saúde mental - que podem funcionar como argumento para a marginalização desse grupo -, é importante mencionar que qualquer trabalhador está sujeito a se afastar do trabalho por problemas de saúde. Inclusive, muitos trabalhadores dos serviços de saúde, que não são apoiadores de pares, se afastam por problemas de saúde mental. Além disso, o esforço necessário para que um apoiador de par volte ao trabalho, não obstante estar passando por um momento difícil do seu processo de recovery, pode servir como exemplo e trazer esperança para os usuários do serviço (Davidson et al., 2012, p. 126), como fica evidente no trecho de relato a seguir:

\begin{abstract}
"Todos nós seres humanos temos dias bons e dias ruins (..) durante a semana, uma ou duas vezes eu fico na baixa. Essa é a minha maior dificuldade, eu, quando eu estou comigo mesmo, chego em casa e fico na baixa. Essa seria a minha dificuldade, "pô, aconteceu isso ou em tal época", esse pensamento já leva a outro, que leva a outro, que acaba me trazendo uma baixa. Então, no meu trabalho no dia-a-dia eu levo o que eu aprendi como um conhecimento, mas dificuldade eu tenho só comigo mesmo, quando estou sozinho vem a dificuldade maior". (Relato do participante 2)
\end{abstract}

Nesse sentido, o impacto do desemprego involuntário, da pobreza e do isolamento é consideravelmente mais oneroso do que os obstáculos com os quais eventualmente o apoiador tenha que lidar no trabalho (Davidson et al., 2012, p. 126). A prática aponta que pessoas em processo de recovery demonstram grande resiliência e persistência, em oposição à suposta "fragilidade" colocada pelo estigma do paciente de saúde mental (Davidson et al., 2012, p. 126). O emprego dos redutores de danos gera renda, senso de pertencimento à comunidade e promove uma rotina organizada a esses trabalhadores, que se identificam enquanto agentes ativos de transformação social.

"Acho que o trabalho ajuda sim, porque todo trabalho ajuda, não só por trabalhar como redutor de danos, mas por ter um emprego fixo e uma rotina de vida". (Relato do participante 2)

"Meu trabalho me garante na saúde mental, me ajuda a cuidar e educar minha filha". (Relato do participante 1) 


\subsection{Adesão ao próprio tratamento}

Os estudos voltados ao suporte de pares colocam com frequência que o compartilhamento de experiências pessoais pode ser um grande desafio para os apoiadores de pares (Davidson et al., 2012, p. 126). Mais uma vez, os benefícios já relatados de aumento da autoestima e um senso positivo de identidade e empoderamento acabam por superar as possíveis dificuldades (Repper et al., 2013, p. 10). Nos relatos dos redutores de danos, o contato com o sofrimento mental dos usuários do serviço e a necessidade de compartilhar suas próprias experiências foram ligados apenas a aspectos positivos de aumento de resiliência e de adesão aos próprios tratamentos.

"Outra coisa que me ajuda, é que vendo a situação dos meninos eu consigo ver o que passei, e consigo fazer algo também pra continuar me tratando. Então, isso me fortalece, para saber que eu tenho que ficar bem mentalmente para cuidar bem das pessoas com quem eu vou ter um convívio (...). Isso me fortalece muito e a partir disso tudo que eu passei eu cheguei à conclusão que eu não posso deixar de tomar meu remédio" (Relato do participante 1)

\section{Influência da experiência vivida anteriormente em sua prática laboral}

\subsection{Linguagem, identificação e empatia}

Para além dos benefícios aos próprios apoiadores de pares, são patentes uma série de incrementos à qualidade do tratamento dos usuários de serviço de saúde mental. A segunda geração de estudos sobre suporte de pares demonstrou que não só os apoiadores de pares podem executar o mesmo trabalho de quem não passou por problemas de saúde mental, mas executam o trabalho com melhores resultados, lançando mão de recursos próprios de alguém que já passou por experiências semelhantes de sofrimento mental. Nesse sentido, apresentam maior empatia e, por vezes, conexão com o usuário do serviço, devido à maior identificação sociocultural em relação a esse sujeito. Tal abordagem diferenciada resulta em maior engajamento de usuários de difícil adesão ao serviço, redução de taxas de internação, redução de dias internados e diminuição de abusos de substâncias (Davidson, 2012, p. 124).

"Como redutor de danos isso veio à tona, porque é uma profissão que depende de uma vivência. Porque eu sei chegar num grupo de uma cena de uso, com várias pessoas usando e eu sei me relacionar 'de boa', sei 'trocar ideia', sou aceito, talvez seja por causa das 'tattoo' que eu tenha no rosto, também, talvez isso possa ajudar um pouco, mas... sou aceito (...) e isso é muito importante para a criação do vínculo... porque a pessoa que está na rua, ela já está em vulnerabilidade e a gente que está ali tentando garantir o direito do próximo, a gente aprende muito com isso". (Relato do participante 1)

"Um paciente que estava fazendo muito uso de cocaína e álcool, a família abandonou ele, e ele um dia pegou fogo na casa dele, os bombeiros quase não viram ele, e aí ele foi pro CERSAM e ajudei a tratar dele. Hoje ele trabalha e está bem, ele vai lá no CERSAM me dar um abraço, esse caso me marcou muito. Então é isso, acreditar na capacidade da pessoa, dizer que é possivel, que aquilo é uma fase, eu trabalho no viés do amor, do respeito e da liberdade dentro da redução de danos e acredito nisso". (Relato do participante 2)

Essas características próprias dos apoiadores de pares são notórias e já foram reconhecidas ainda no século XVIII por Jean Baptiste Pussin em carta a Philippe Pinel ao descrever sua preferência por contratar pessoas que já tinham histórico de sofrimento mental para trabalharem no hospital do qual era superintendente em Paris, devido a considerá-las "usualmente mais gentis, honestas e humanas" (Weiner, 1979). Por já terem estado em situações semelhantes, os apoiadores de pares conseguem estabelecer relações mais sólidas com os usuários, baseadas em empatia e preocupação autêntica. Além disso, não focam apenas na doença em si, mas no auxílio para outras necessidades básicas do dia-a-dia do usuário do serviço (Davidson et al., 2018, p. 11).

\begin{abstract}
"Teve um ocorrido comigo antes de sair de férias. Nós do consultório de rua, primeiro a gente tem que chegar, criar um laço, e depois se ele topar, a gente leva até o serviço. Nesse dia, fizemos tudo isso, e aí levamos no CMT, só que ele estava muito debilitado, urinando e defecando na roupa, ele precisava de um banho... nossa, pra ele tomar esse banho, você não imagina, falaram que passou no horário do banho, como ele vai entrar todo sujo desse jeito? Fui em uma, em outra, ninguém resolvia. Fui na gerente. 'Uai, você teve dificuldade de organizar isso', ela disse. 'Vai em tal pessoa e pega a toalha'. Mas precisei da gerente. Eu acho que o pessoal tem que ter um pouco de sensibilidade, quem vai trabalhar com essa galera de situação precária, a pessoa já está debilitada psicologicamente, e aí quando chega até o equipamento, a gente tem que fazer ser legal pra ele, chegou no serviço ainda ser maltratado [não dá]. Vejo a dificuldade, tanto como usuário, quanto como profissional do serviço, vejo nos outros profissionais, parece que eles não queriam nem estar ai trabalhando". (Relato do participante 1)
\end{abstract}

\subsection{Conhecimento próprio sobre os efeitos e condução dos tratamentos}

Nesse ponto, é importante salientar que não é a função dos apoiadores de pares e mesmo dos profissionais que não são apoiadores de pares "tratar" a doença mental. Em verdade, em um serviço orientado pelo recovery busca-se reestabelecer a esperança do usuário, auxiliá-lo a acessar da melhor maneira os dispositivos da rede de saúde pública - não apenas os dispositivos de saúde mental - e empoderá-los para que pratiquem autocuidado e atos de cidadania. Logo, em momento algum o foco deve estar na "cura" da doença, mas sim na melhora da qualidade de vida e reinserção na comunidade (Davidson et al., 2018, p.12). Por suas experiências prévias e a vivência de seu próprio processo de recovery, os apoiadores de pares tendem a reconhecer o problema do usuário do serviço com maior assertividade e rapidez do que outros profissionais. Essa conexão baseada na empatia, em conjunto com o fato de estarem em um estágio mais avançado do recovery, acaba por estabelecer uma relação em que o apoiador deposita expectativas positivas e acredita na possibilidade do recovery do paciente assistido (Davidson, 2012, p. 124). Tal abordagem se diferencia daquelas 
baseadas no estigma de que os pacientes em saúde mental são irrecuperáveis, o que evita medidas como recorrentes internações coercitivas e outras práticas que não são eficazes e aumentam o custo dos serviços públicos de saúde mental (Davidson, 2016, p. 1094).

\begin{abstract}
"Cheguei lá, me enturmei com a meninada (...) Chegou um adolescente que o equipamento achava que era difícil de lidar (...) Eu passei a tarde inteira (...) conversando com esse adolescente (...) brincando com ele, jogando bola, interagindo com ele. Aí chegou a hora de ele tomar o remédio. Ninguém fazia ele tomar o remédio, porque ele não queria tomar o remédio, porque ele era muito novo para tomaro remédio, ele não queria tomar o remédio. Aí eu 'chamei ele' 'de quebrada', longe de todo mundo, só eu e ele. 'Cheguei para ele' e falei: '(...) Qual o problema, 'mano', de tomar os comprimidos? E tudo mais... por que você está resistindo tanto?' Aí ele: 'Não, tio, eu não vou tomar isso não porque isso me dá outros problemas no corpo e eu não quero tomar isso não porque eu fico 'grogue'. Eu falei: 'Pois é, mas você já falou isso tudo com seu médico, com seu psiquiatra, tudo o que você sente quando você toma essa medicação, o que realmente você está sentindo? Faz isso!" Mas toma o remédio primeiro, já que está prescrito para você tomar é necessário você tomar...' Então, a minha vivência, o que eu sei, é importante o remédio na vida de quem precisa... 'fez ele' rever o conceito dele a respeito e naquele momento ali tomar o remédio. Isso foi um grande avanço para mim, uma vitória no dia" (Relato do participante 1).
\end{abstract}

\begin{abstract}
“O CERSAM que fiz tratamento, esteve 'perdido' por muito tempo em função da droga, e quando você está assim com certeza não está com saúde mental íntegra. E hoje há a minha facilidade pra tratar, porque fui tratado com o mesmo problema, então sempre acredito na recuperação e na capacidade da pessoa" (Relato do participante 1).
\end{abstract}

\subsection{Próprias experiências como fonte de esperança}

Nessa perspectiva de reestabelecer a esperança do usuário do serviço em seu processo de recovery, o apoiador de pares acaba por incitar a esperança a partir da sua própria jornada de recovery. Elemento essencial dentro dessa jornada é o fato de que, além de ter conseguido lidar, por meio de diferentes estratégias, com seu problema de saúde mental, o apoiador de pares se empoderou e se reinseriu na sociedade pelo próprio fato de ter sido contratado como apoiador de par, tendo um trabalho remunerado, uma rotina estruturada e uma importantíssima função social (Davidson, 2012, p. 124).

“(...) Às vezes eu paro assim, olho para ele [usuário] e falo: 'Ô, mano, eu tomo remédio controlado até hoje'. Aí na hora já vem a aceitação da pessoa, a pessoa: 'E aí, cara, como que é? Você toma o remédio e trabalha, tudo mais? ' Porque tem um preconceito com remédio, todo mundo tem, ainda mais o mental. Porque doença mental é diferente. As pessoas ficam diferentes do que nas outras doenças" (Relato do participante 2).

\section{Discussão e conclusões}

Verifica-se, pormeio da análise dos relatos compilados na seção anterior, que graças às suas experiências prévias e à vivência de seu próprio processo de recovery, os redutores de danos em Belo Horizonte tendem a oferecer um trabalho análogo ao suporte de pares. Nessa cidade, o trabalho dos redutores de danos é uma importante tarefa adaptada à realidade do território. Sendo assim, a devida inserção do redutor de danos na comunidade que abordará é importante característica para o trabalho em redução de danos. A atuação profissional dos redutores de danos ocorre no âmbito dos múltiplos dispositivos de saúde da região em questão, bem como em diversos outros locais do território, tais como espaços de cultura, educação, esportes e lazer, tal como verificado nos relatos pertinentes ao presente estudo.

Nas ruas, os redutores de danos percorrem o território em unidades móveis, localizando cenas de uso de álcool e outras substâncias psicoativas e iniciando conversas empáticas com as pessoas encontradas, que culminam, por vezes, no encaminhamento bem-sucedido de um usuário fragilizado a uma unidade de saúde. Além disso, os redutores de danos oferecem insumos, promovem atividades culturais como alternativas ao uso de substâncias e fazem o transporte de usuários para atendimentos em saúde, bem como para espaços de esportes, cultura e lazer. Aparece, nos relatos documentados, a valorização das experiências prévias dos redutores de danos como fonte crucial de conhecimento para o desenvolvimento de estratégias objetivando melhor atendimento.

Se reconhecidas como suporte de pares, e, consequentemente, como estratégia de recovery, as medidas de redução de danos poderiam ter melhor destacados seus êxitos no atendimento a demandas identificáveis na rede de saúde mental de Belo Horizonte. Dentre tais êxitos podem ser listados: aproximação entre profissionais e usuários, diminuição do estigma, recuperação de esperança, visão mais empática no cuidado, exercício da cidadania e cultivo da autonomia dos participantes e responsabilização pela vida - tanto pela própria, quanto pela daqueles da comunidade ao seu redor.

Diante das experiências exitosas corroboradas por estudos sobre o suporte de pares, é oportuno fortalecer o vínculo empregatício e dar melhores condições de trabalho aos redutores de danos, além de ampliar a proposta de suporte de pares para outros serviços da rede de saúde mental brasileira. Espera-se que o presente artigo consista em material para o fomento, o fortalecimento e a formação de organizações voltadas à redução de danos e ao suporte de pares como estratégia de recovery, bem como para subsidiar o debate e incentivar as necessárias investigações futuras envolvendo a temática.

\section{Referências bibliográficas}

Antunes, A. (2019) Redução de danos no fio da navalha. Escola Politécnica de Saúde João Venâncio/Fiocruz. http://www.epsjv.fiocruz.br/noticias/ reportagem/reducao-de-danos-no-fio-da-navalha

APA - American Psychiatric Association. (n.d.). Recovery Oriented Care. https://www.psychiatry.org/psychiatrists/practice/professionalinterests/recovery-oriented-care 
Braun, V., \& Clarke, V. (2013). Sucessful Qualitative Resourse: A practical Guide for beginers. SAGE Publications. https://www.researchgate. net/publication/256089360 Successful Qualitative Research A Practical Guide for Beginners

Baccari, I. O. P., Onocko, R. T., Stefanello, S. (2015). Recovery: revisão sistemática de um conceito. http://www.scielo.br/pdf/csc/v20n1/14138123-csc-20-01-00125.pdf

Campos, F. A. L., Sousa, A. R. P., Rodrigues, V. P. da C., Marques, A. J. P. da S., Dores, A. A. M. da R., Queirós, C. M. L. (2014). Peer support for people with mental illness. Archives of Clinical Psychiatry, 41(2), 49-55. https://doi.org/10.1590/0101-60830000000009

Costa, M.; Baker, M.; Davidson, L. (2017). Provider perspectives on employment for people with serious mental illness. International Journal of Social Psychiatry, 63(7), 632-640. https://doi. org/10.1177\%2F0020764017725769

Costa, M. (2017). Recovery como estratégia para avançar a reforma psiquiátrica no Brasil. Cadernos Brasileiros de Saúde Mental. https:// doi.org/10.5007/cbsm.v9i21.69532

Davidson, L. (2016). The recovery movement: Implications for mental health care and enabling people to participate fully in life. Health Affairs, 35(6), 1091-1097. https://doi.org/10.1377/hlthaff.2016.0153

Davidson, L. (2003). Living Outside Mental Illness. Qualitative studies of recovery in Schizophrenia. New York University Press. https://doi. org/10.1176/appi.ps.55.9.1073-a

Davidson, L.; Bellamy, C.; Chinman, M.; Farkas, M.; Ostrow, L.; Cook, J.; Jonikas, J.; Rosenthal, H.; Bergeson, S.; Daniels, A.; Salzer, M. (2018). Revisiting the rationale and evidence for peer support. Psychiatry Times, 35(6), 1-3. https://openexcellence.org/revisiting-rationaleevidence-peer-support/

Davidson, L. (2012). Peer support among persons with severe mental illnesses: a review of evidence and experience. World Psychiatry. https://doi.org/10.1016/j.wpsyc.2012.05.009

Davidson, L.; Chinman, M.; Sells, D.; Rowe, M. (2006). Peer support among adults with serious mental illness: a report from the field. Schizophr Bull. 32(3), 443-450. https://doi.org/10.1093/schbul/sbj043

DMHAS - Department Of Mental Health And Addiction Service. (2002). Recovery Resource Guide.https://portal.ct.gov/-/media/DMHAS/ Recovery/resourceguidepdf.pdf?la=en

Nardi, H. C., \& Rigoni, R. de Q. (2005). Marginalidade ou cidadania? a rede discursiva que configura o trabalho dos redutores de danos. Psicologia em Estudo, 10(2), 273-282. https://doi.org/10.1590/S141373722005000200014
Onocko-Campos, R. T.; Palombini, A. de L.; Silva, A. do E.; Passos, E.; Leal, E. M.; Serpa J. O. D.; Marques, C. de C.; Gonçalves, L. L. M.; Santos, D. V. D.; Passos, E. H.; \& Souza, T. P. (2011). Redução de danos e saúde pública: construções alternativas à política global de "guerra às drogas". Psicologia \& Sociedade, 23(1), 154-162. http://dx.doi. org/10.1590/S0102-71822011000100017

Onocko-Campos, R.; Palombini, A.; Silva, A.; Passos, E.; Leal, E.; Domont, O. de S.; Marques, C.; Gonçalves, L.; Santos, D.; Surjus, L.; Arantes, R.; Emerich, B.; Otanari, T.; Stefanello, S. (2012). Multicenter adaptation of the guide Autonomous management of medication. Interface: Communication, Health, Education. 16(43), 967-980. https://www. scielosp.org/article/icse/2012.v16n43/967-980/en/

Petuco, D. R. da S. (2014). Redução de Danos: das técnicas à ética do cuidado. In: RAMMINGER, Tatiana; SILVA, Martinho (Org.). Mais substâncias para o trabalho em saúde com usuários de drogas. Rede Unida. https://www.researchgate.net/profile/Marcelo-Dalla-Vecchia/ publication/321873266_O_apoio_institucional_na_implantacao_ de_estrategias_de_reducao_de_danos_por_que_e_para_quel links/5a37231a0f7e9b10d8484e56/0-apoio-institucional-naimplantacao-de-estrategias-de-reducao-de-danos-por-que-e-paraque.pdf

Petuco, D. R. da S. (2011). Redução de danos. In: Álcool e outras drogas. Conselho Regional de Psicologia de São Paulo.

Silveira, Ricardo Wagner Machado da. (2016). Redução de danos e acompanhamento terapêutico: aproximações possíveis. Revista do NUFEN, 8(1), 110-128. http://pepsic.bvsalud.org/scielo.php?script=sci arttext\&pid $=\$ 2175-25912016000100008 \& \operatorname{lng}=p t \& t \operatorname{lng}=p t$

Repper, J. (2013). ROC briefing paper 7. Peer support workers: a practical guide to implementation. Centre for Mental Health. http://citeseerx.ist. psu.edu/viewdoc/download?doi=10.1.1.688.7633\&rep=repl\&type=pdf

Tondora, J; Heerema; Delphin; Andres-Hyman; O'connel, M.; \& Davidson, L. (2008).Practice Guidelines for Recovery-Oriented Care for Mental Health and Substance Use Conditions. Connecticut Department of Mental Health and Addiction Services. http://www.aamentalhealth. org/aaco/docs/recovery practice guidelines-connecticut.pdf

Weiner, D. B. (1979). The apprenticeship of Philippe Pinel: a new document, "Observations of Citizen Pussin on the Insane". Am J Psychiatry, (36), 1128-1134. https://doi.org/10.1176/ajp.136.9.1128 


\section{Apêndice}

\section{Agora}

- Não dá pra esperar?

- Nem um segundo a mais!

- Nem com música?

- Tá, eu escolho, me empresta o celular.

- Só se não deixar cair nem tocar as braba!

- Fechou, mas ainda vamos lá fora.

- Só não vai ser agora.

- E se eu começar a angustiar, não conseguir colocar em palavras, transbordar? precisar desaguar em outro lugar

-Tá ouvindo, tio?

O som das ondas quebrando nas portas,

das Marés aumentando no pátio,

das grandes Bacias elevando no leito

Posso ensinar vocês a nadar,

posso também ensinar vocês a observar

O mar

que quase sempre, nos mínimos detalhes,

avisa que vai agitar.

Ondas, tempestades, maremotos e tufão,

quase sempre se contêm com uma boa conversa,

um atencioso e delicado observar, escutar, acolher.

Já vi pré-terremoto terminar em brincadeiras no chão,

possíveis tufões se dissipando em rimas e bailão.

Maremotos se banhando em baldes para refrescar,

furacão em céu aberto empinando pipa.

Pensa só, quem diria?

Entre tempestade e furacão,

nos mantemos, firmes e fortes.

Efetivando o cuidado

e a política da redução:

de danos

de vulnerabilidades

de riscos.

Acessando a cidade, criando possibilidades,

compartilhando informação,

possibilitando o brincar.

Fazendo disso a principal ferramenta

do Agora.

(Louise Arcanjo/ Redutora de Danos CERSAMI-NE) 